\title{
Simulations and performance of the QUBIC optical beam combiner
}

\author{
C. O'Sullivan, D. Burke, D. Gayer, J. D. Murphy, S. \\ Scully, et al.
}

C. O'Sullivan, D. Burke, D. Gayer, J. D. Murphy, S. Scully, M. De Leo, M. De Petris, A. Mattei, A. Zullo, A. Mennella, M. Zannoni, N. Bleurvacq, C. Chapron, J.-Ch. Hamilton, M. Piat, P. Ade, G. Amico, D. Auguste, J. Aumont, S. Banfi, G. Barbarán, P. Battaglia, E. Battistelli, A. Baù, B. Bélier, D. Bennett, L. Bergé, J.-Ph. Bernard, M. Bersanelli, M.-A. Bigot-Sazy, J. Bonaparte, J. Bonis, G. Bordier, E. Bréelle, E. Bunn, D. Buzi, A. Buzzelli, F. Cavaliere, P. Chanial, R. Charlassier, F. Columbro, G. Coppi, A. Coppolecchia, F. Couchot, R. D'Agostino, G. D'Alessandro, P. de Bernardis, G. De Gasperis, A. Di Donato, L. Dumoulin, A. Etchegoyen, A. Fasciszewski, C. Franceschet, M. M. Gamboa Lerena, B. García, X. Garrido, M. Gaspard, A. Gault, M. Gervasi, M. Giard, Y. Giraud-Héraud, M. Gómez Berisso, M. González, M. Gradziel, L. Grandsire, E. Guerrard, D. Harari, V. Haynes, S. Henrot-Versillé, D. T. Hoang, F. Incardona, E. Jules, J. Kaplan, A. Korotkov, C. Kristukat, L. Lamagna, S. Loucatos, T. Louis, A. Lowitz, V. Lukovic, R. Luterstein, B. Maffei, S. Marnieros, S. Masi, A. May, M. McCulloch, M. C. Medina, L. Mele, S. Melhuish, L. Montier, L. M. Mundo, J. A. Murphy, E. Olivieri, A. Paiella, F. Pajot, A. Passerini, H. Pastoriza, A. Pelosi, C. Perbost, O. Perdereau, F. Pezzotta, F. Piacentini, L. Piccirillo, G. Pisano, G. Polenta, D. Prêle, R. Puddu, D. Rambaud, P. Ringegni, G. E. Romero, M. Salatino, A. Schillaci, C. G. Scóccola, S. Spinelli, M. Stolpovskiy, F. Suarez, A. Tartari, J.-P. Thermeau, P. Timbie, S. A. Torchinsky, M. Tristram, V. Truongcanh, C. Tucker, G. Tucker, S. Vanneste, D. Viganò, N. Vittorio, F. Voisin, B. Watson, F. Wicek, "Simulations and performance of the QUBIC optical beam combiner," Proc. SPIE 10708, Millimeter, Submillimeter, and Far-Infrared Detectors and Instrumentation for Astronomy IX, 107082I (9 July 2018); doi: $10.1117 / 12.2313256$

Event: SPIE Astronomical Telescopes + Instrumentation, 2018, Austin, Texas, United States 


\title{
Simulations and performance of the QUBIC optical beam combiner
}

\author{
C. O'Sullivan*a , D. Burke ${ }^{\mathrm{a}}$, D. Gayer ${ }^{\mathrm{a}}$, J. D. Murphy ${ }^{\mathrm{a}}$, S. Scully ${ }^{\mathrm{a}, \mathrm{b}}$, M. De Leo ${ }^{\mathrm{c}}$, \\ M. De Petris ${ }^{\mathrm{c}, \mathrm{d}}$, A. Mattei ${ }^{\mathrm{d}}$, A. Zullo ${ }^{\mathrm{d}}$, A. Mennella ${ }^{\mathrm{e}, \mathrm{f}}$, M. Zannoni ${ }^{\mathrm{g}, \mathrm{h}}$, N. Bleurvacq ${ }^{\mathrm{i}}$,

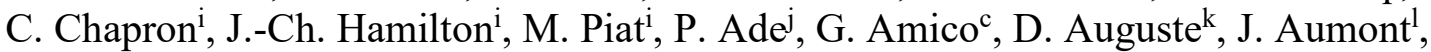
S. Banfi ${ }^{\mathrm{g}, \mathrm{h}}$, G. Barbarán ${ }^{\mathrm{m}}$, P. Battaglia ${ }^{\mathrm{e}}$, E. Battistelli ${ }^{\mathrm{c}, \mathrm{d}}$, A. Baù ${ }^{\mathrm{g}, \mathrm{h}}$, B. Bélier ${ }^{\mathrm{n}}$, D. Bennett ${ }^{\mathrm{a}}$, L. Bergéo, J.-Ph. Bernard ${ }^{p}$, M. Bersanelli ${ }^{\text {eff }}$, M.-A. Bigot-Sazy ${ }^{\mathrm{i}}$, J. Bonaparte ${ }^{\mathrm{q}}$, J. Bonis ${ }^{\mathrm{k}}$, G. Bordieri, E. Bréelle ${ }^{\mathrm{i}}$, E. Bunn ${ }^{\mathrm{r}}$, D. Buzic ${ }^{\mathrm{i}}$, A. Buzzelli ${ }^{\mathrm{s}}$, F. Cavaliere ${ }^{\mathrm{e}}$, P. Chanial, R. Charlassier ${ }^{\mathrm{i}}$, F. Columbro ${ }^{\mathrm{c}}$, G. Coppi $\mathrm{i}^{\mathrm{t}}$, A. Coppolecchia ${ }^{\mathrm{c}, \mathrm{d}}$, F. Couchot ${ }^{\mathrm{k}}$, R. D'Agostino, G. D’Alessandro ${ }^{\text {,dd }}$, P. de Bernardis ${ }^{\text {c,d }}$, G. De Gasperis ${ }^{\mathrm{s}}$, A. Di Donato ${ }^{\mathrm{q}}$, L. Dumoulin ${ }^{\circ}$, A. Etchegoyen ${ }^{\mathrm{u}}$, A. Fasciszewski ${ }^{\mathrm{q}}$, C. Franceschet ${ }^{\mathrm{e}, \mathrm{f}}$, M. M. Gamboa Lerena ${ }^{\mathrm{v}}$, B. García ${ }^{\mathrm{u}}$, X. Garrido ${ }^{\mathrm{k}}$, M. Gaspard ${ }^{\mathrm{k}}$, A. Gault ${ }^{\mathrm{w}}$, M. Gervasi ${ }^{\mathrm{g}, \mathrm{h}}$, M. Giard ${ }^{\mathrm{p}}$, Y. Giraud-Héraud ${ }^{\mathrm{i}}$, M. Gómez Berisso ${ }^{\mathrm{x}}$, M. González ${ }^{\mathrm{x}}$, M. Gradziel ${ }^{\mathrm{a}}$, L. Grandsire ${ }^{\mathrm{i}}$, E. Guerrard ${ }^{\mathrm{k}}$, D. Harari ${ }^{\mathrm{x}}$, V. Haynes ${ }^{\mathrm{t}}$, S. Henrot-Versillé ${ }^{\mathrm{k}}$, D. T. Hoang ${ }^{\mathrm{i}}$, F. Incardona, ${ }^{\mathrm{e}, \mathrm{f}}$, E. Jules ${ }^{\mathrm{k}}$, J. Kaplan, A. Korotkov ${ }^{\mathrm{y}}$, C. Kristukat ${ }^{\mathrm{z}}$, L. Lamagna ${ }^{\mathrm{c}, \mathrm{d}}$, S. Loucatos ${ }^{\mathrm{i}}$, T. Louis ${ }^{\mathrm{k}}$, A. Lowitz ${ }^{\mathrm{w}}$, V. Lukovic ${ }^{\mathrm{s}}$, R. Luterstein ${ }^{\mathrm{m}}$, B. Maffei ${ }^{1}$, S. Marnieros ${ }^{\mathrm{o}}, \mathrm{S}$. Masi ${ }^{\mathrm{c}, \mathrm{d}}$, A. May ${ }^{\mathrm{t}}, \mathrm{M} . \mathrm{McCulloch}^{\mathrm{t}}$, M. C. Medina ${ }^{\text {aa }}$, L. Mele ${ }^{c}$, S. Melhuish ${ }^{\text {t }}$ L. Montier ${ }^{\mathrm{p}}$, L. M. Mundo ${ }^{\text {ab }}$, J.A. Murphy, E. Olivierio , A. Paiella ${ }^{\mathrm{c}, \mathrm{d}}$, F. Pajot ${ }^{\mathrm{p}}$, A. Passerini ${ }^{\mathrm{g}, \mathrm{h}}$, H. Pastoriza ${ }^{\mathrm{x}}$, A. Pelosi ${ }^{\mathrm{d}}$, C. Perbost ${ }^{\mathrm{i}}$, O. Perdereau ${ }^{\mathrm{k}}$, F. Pezzotta ${ }^{\mathrm{e}}$, F. Piacentini ${ }^{\mathrm{c}, \mathrm{d}}$, L. Piccirillo ${ }^{\mathrm{t}}$, G. Pisano ${ }^{\mathrm{j}}$, G. Polenta ${ }^{\mathrm{c}, \mathrm{d}}$, D. Prêle ${ }^{\mathrm{i}}$, R. Puddu ${ }^{\text {c,d }}$, D. Rambaud ${ }^{\text {, P. Ringegni }}{ }^{\text {ab }}$, G. E. Romero ${ }^{\text {aa }}$, M. Salatino, A. Schillaciac, C. G. Scóccola ${ }^{v}$, S. Spinelli ${ }^{\mathrm{g}}$, M. Stolpovskiyi, F. Suarez ${ }^{\mathrm{u}}$, A. Tartari', J.-P. Thermeau,

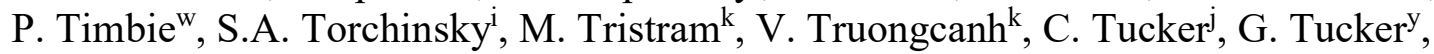

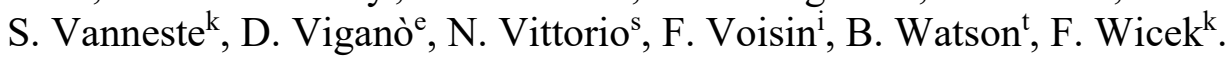

${ }^{a}$ Dept. of Exp. Physics, National University of Ireland, Maynooth, Co. Kildare, Ireland; ${ }^{b}$ Institute of Technology Carlow, Co. Carlow, Ireland; 'Dip. Fisica, Università di Roma "La Sapienza", Roma,

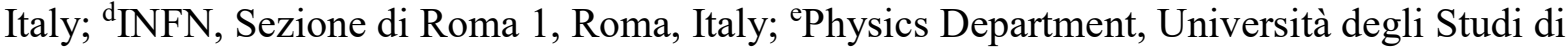
Milano, Milano, Italy; ${ }^{\mathrm{f}}$ INFN Milano, Milano, Italy; ${ }^{\mathrm{P}} \mathrm{Physics}$ Department, Università di Milano -

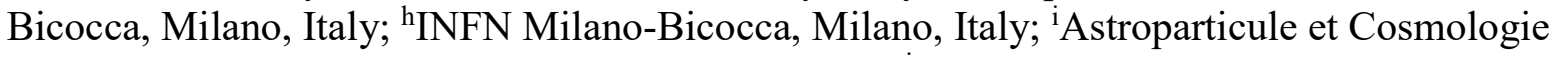
(CNRS-IN2P3), Université Paris Diderot-Paris 7, France; ${ }^{j}$ School of Physics and Astronomy, Cardiff University, UK; ${ }^{k}$ Laboratoire de l'Accélérateur Linéaire (CNRS-IN2P3), Orsay, Paris, France;

${ }^{1}$ Institut d'Astrophysique Spatiale (CNRS-INSU), Orsay, Paris, France; ' ${ }^{\text {Regional Noroeste }}$ (CNEA), Salta Cuty, Salta Province, Argentina; ${ }^{\mathrm{n}}$ Centre de Nanosciences et de Nanotechnologies, Orsay, Paris, France; ${ }^{\circ}$ Centre de Spectrométrie Nucléaire et de Spectrométrie de Masse (CNRSIN2P3), Orsay, Paris, France; ${ }^{\mathrm{p} I n s t i t u t ~ d e ~ R e c h e r c h e ~ e n ~ A s t r o p h y s i q u e ~ e t ~ P l a n e ́ t o l o g i e ~(C N R S-~}$ INSU), Toulouse, France; ${ }^{q}$ Centro Atómico Constituyentes (CNEA), Buenos Aires, Argentina;

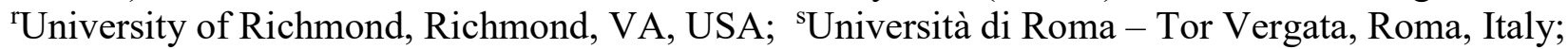
${ }^{\text {tS }}$ chool of Physics \& Astronomy, University of Manchester, UK; "Instituto de Tecnologías en Detección y Astropartículas (CNEA, CONICET, UNSAM), Buenos Aires, Argentina; ${ }^{\vee}$ Facultad de

Cs Astronómicas y Geofísicas (FCAG-UNLP/CONICET), Universidad Nacional de La Plata, Argentina; ${ }^{\text {w} U n i v e r s i t y ~ o f ~ W i s c o n s i n, ~ M a d i s o n, ~ W I, ~ U S A ; ~}{ }^{x}$ Centro Atómico Bariloche and Instituto Balseiro (CNEA), Río Negro, Argentina ${ }^{y}$ Brown University, Providence, RI, USA; ${ }^{z}$ Escuela de

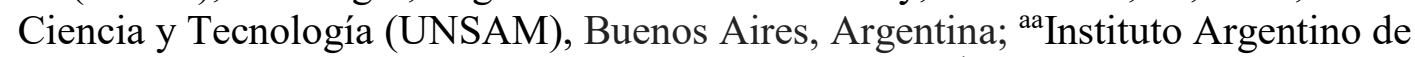
Radioastronomía (CONICET, CIC), Buenos Aires, Argentina; ${ }^{\text {ab }}$ GEMA, Universidad Nacional de

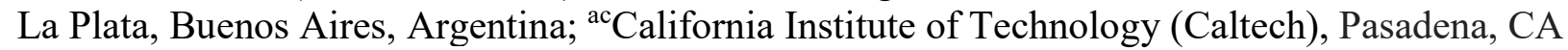
91125, USA, *corresponding author: creidhe.osullivan@mu.ie

\footnotetext{
Millimeter, Submillimeter, and Far-Infrared Detectors and Instrumentation for Astronomy IX, edited by Jonas Zmuidzinas, Jian-Rong Gao, Proc. of SPIE Vol. 10708, 107082I (c) 2018 SPIE · CCC code: 0277-786X/18/\$18 - doi: 10.1117/12.2313256
} 


\begin{abstract}
QUBIC, the Q \& U Bolometric Interferometer for Cosmology, is a novel ground-based instrument that aims to measure the extremely faint B-mode polarisation anisotropy of the cosmic microwave background at intermediate angular scales (multipoles of $l=30-200$ ). Primordial B-modes are a key prediction of Inflation as they can only be produced by gravitational waves in the very early universe. To achieve this goal, QUBIC will use bolometric interferometry, a technique that combines the sensitivity of an imager with the immunity to systematic effects of an interferometer. It will directly observe the sky through an array of back-to-back entry horns whose beams will be superimposed using a cooled quasioptical beam combiner. Images of the resulting interference fringes will be formed on two focal planes, each tiled with transition-edge sensors, cooled down to $320 \mathrm{mK}$. A dichroic filter placed between the optical combiner and the focal planes will select two frequency bands (centred at $150 \mathrm{GHz}$ and $220 \mathrm{GHz}$ ), one frequency per focal plane. Polarization modulation will be achieved using a cold stepped half-wave plate (HWP) and polariser in front of the sky-facing horns.

The full QUBIC instrument is described elsewhere ${ }^{1,2,3,4}$; in this paper we will concentrate in particular on simulations of the optical combiner (an off-axis Gregorian imager) and the feedhorn array. We model the optical performance of both the QUBIC full module and a scaled-down technological demonstrator which will be used to validate the full instrument design. Optical modelling is carried out using full vector physical optics with a combination of commercial and in-house software. In the high-frequency channel we must be careful to consider the higher-order modes that can be transmitted by the horn array. The instrument window function is used as a measure of performance and we investigate the effect of, for example, alignment and manufacturing tolerances, truncation by optical components and off-axis aberrations. We also report on laboratory tests carried on the QUBIC technological demonstrator in advance of deployment to the observing site in Argentina.
\end{abstract}

Keywords: CMB, B-modes, bolometric interferometry, QUBIC, physical optics

\title{
1. INTRODUCTION
}

\subsection{QUBIC and the cosmic microwave background}

The Q \& U Bolometric Interferometer for Cosmology (QUBIC) ${ }^{1,2}$ is a ground-based experiment that is designed to measure very faint polarisation anisotropies in the cosmic microwave background (CMB) radiation. This polarisation anisotropy is generally decomposed into E-modes (curl-free) and B-modes (divergence-free). Density (scalar) perturbations in the early Universe give rise to the dominant E-mode polarisation while any gravitational waves (tensor perturbations) present also give rise to B-modes. QUBIC aims to measure these faint primordial B-mode polarisation anisotropies, a key prediction of Inflation theory 5 . Unfortunately, primordial B-modes must be measured against a strong foreground of instrumental, atmospheric and galactic sources as well as the conversion of E-modes into B-modes by intervening largescale structure in the Universe; primordial B-modes have yet to be detected. QUBIC aims to constrain the tensor-to-scalar ratio, $r$, by exploiting the sensitivity of bolometers combined with the control of systematic errors offered by interferometry and will target intermediate angular scales around the $l \approx 100$ recombination peak in the B-mode angular power spectrum. The QUBIC project is described in more detail elsewhere in these proceedings ${ }^{3}$.

The first QUBIC module (hereafter referred to as the full instrument, FI) will operate as a dual-band instrument (at 150 $\mathrm{GHz}$ and $220 \mathrm{GHz}$ ) and will comprise 400 back-to-back horns (12.9 $\mathrm{FWHM}$ ) whose signals will be added using a quasioptical combiner. The resulting interference fringes will be imaged on two cooled ( $320 \mathrm{mK})$ arrays of $1024 \mathrm{TES}$ bolometers (one focal plane per frequency band) in order to achieve background-limited sensitivity. A dichroic filter will be used to split the radiation and direct it towards two focal planes placed at right angles to one another: the $150 \mathrm{GHz}$ band is transmitted while the $220 \mathrm{GHz}$ is reflected. A rotating half-wave plate and a fixed polariser in front of the sky-facing horns will be used to directly construct synthetic images of the $I, Q$ and $U$ Stokes parameters observed through the instrument primary beam. Unlike other ground-based experiments, all the QUBIC mirrors are inside the cryostat $(4 \mathrm{~K})$ and after the polariser in the optical chain, reducing the effect of any instrumental polarisation due to them. An autocalibration technique, making use of redundant baselines, has been developed ${ }^{6,7}$ so that QUBIC will achieve unprecedented control of systematics along with a sensitivity comparable to that of more traditional imaging polarimeters. Simulations have shown that this first module could constrain the tensor-to-scalar ratio down to $\sigma(r)=0.01$ after a two-year survey ${ }^{4}$. If successful, it is hoped to add further QUBIC modules in the future. 
As an intermediate step, it was decided to build a QUBIC technological demonstrator (TD) to validate the full instrument design and test it electrically, thermally and optically. The TD differs from the FI in that it has a reduced number of detector pixels (256 150-GHz pixels i.e. one quarter of one focal plane), a reduced number of horns and switches (an $8 \times 8$ array rather than $20 \times 20$ ), reduced mirror size $(400 \mathrm{~mm}$ in diameter rather than $600 \mathrm{~mm}$ ), reduced filter sizes (up to 280 $\mathrm{mm}$ in diameter) and a neutral density filter instead of a dichroic. The scaling-down of the instrument for the demonstrator means that the mirrors, filters and horn array could be manufactured in the machine shops of collaborating institutes. The TD is currently undergoing integration and testing at the Laboratoire Astroparticule \& Cosmologie (APC) in Paris.

\subsection{Layout of the paper}

This paper is organized as follows: we present the design of the QUBIC optical beam combiner (Section 2) and the range of simulation techniques used to analyse and characterise its performance (Section 3). In Section 4 the manufacturing and performance of the real mirrors are discussed before finishing with their alignment and testing in Section 6 and future plans in Section 7.

\section{QUBIC OPTICAL BEAM COMBINER}

\subsection{The beam combiner}

QUBIC operates as a Fizeau interferometer: the beams from an array of back-to-back horns at the entrance aperture are superimposed on a focal plane by the beam combiner. The combiner is essentially an imager since the chief ray from each horn is focussed at the centre of the focal plane. Each pair of horns produces a fringe pattern on the focal plane and, for perfect imaging, equivalent baselines produce identical fringe patterns. The fringe pattern image is sampled by an array of bolometers (bolometric interferometry). Figure 1 shows an example of an ideal imager made using two paraxial lenses.

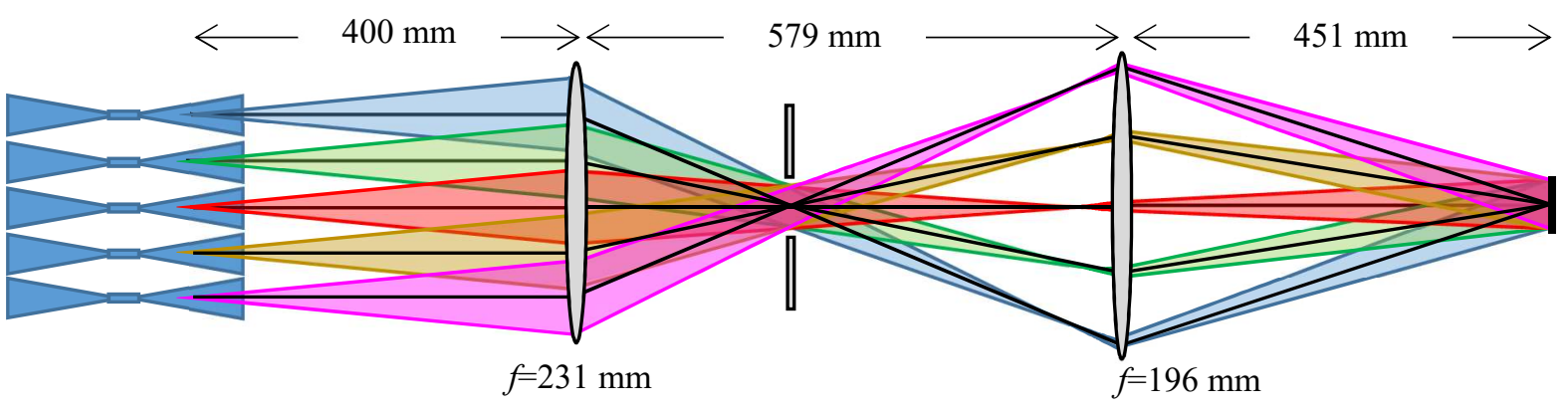

Figure 1. An ideal imager superimposing the beams from an input array of horns. (Ray tracing in Zemax software was used to produce the beams in this diagram.) This optical system is the on-axis equivalent of the QUBIC combiner, with a final effective focal length equal to $300 \mathrm{~mm}$.

The layout of the input horn array for both the FI and TD are shown in Figure 2. Each element of the array consists of a back-to-back pair of conical corrugated horns made from a series of stacked platelets with holes of varying radii. The horns have been designed to transmit a single mode at $150 \mathrm{GHz}$ (giving a farfield beam pattern of $12.9^{\circ} \mathrm{FWHM}$ ), however up to five modes can propagate in the upper 220-GHz band. A more detailed discussion of the horn design and performance can be found in previous papers $8,8,10$.

Waveguide switches placed between the horns in each back-to-back pair will allow signals from individual horns or baselines to be turned on and off. The switches will be opened and closed regularly while QUBIC observes a calibration source to allow self-calibration of the system ${ }^{6,7}$. QUBIC will be used as a synthetic imager, observing the fringes from all baselines simultaneously. If the horns used in each back-to-back pair are identical, then the PSF for an on-axis source (its image on the focal plane) is identical to the synthesised beam for a detector in the centre of the focal plane (i.e. the response of the detector to a point source at different angles on the sky, neglecting for the moment the finite size of the detector). The same is true for the PSF for an off-axis point on the sky and the synthesised beam for the corresponding point on the 
focal plane. Figure 3 ((a)-(c)) shows the focal plane images for a single short baseline, a single long baseline and all baselines simultaneously for such an imager. The combined fringe pattern (Figure 3(c)) is simply an image of the sky (here an on-axis point source) convolved with the synthesised beam of the instrument (for comparison, the synthesised beam of an imaging telescope would only have the on-axis peak). This synthesised beam, at a single wavelength, is largely determined by the location of the horns in the input array. The field-of-view of the instrument on the sky $\left(12.9^{\circ}\right)$ is determined by their beam pattern. The horn beam pattern on the focal plane is the envelope of the fringe pattern in Figure 3(a) and (b) (see also Figure 13).

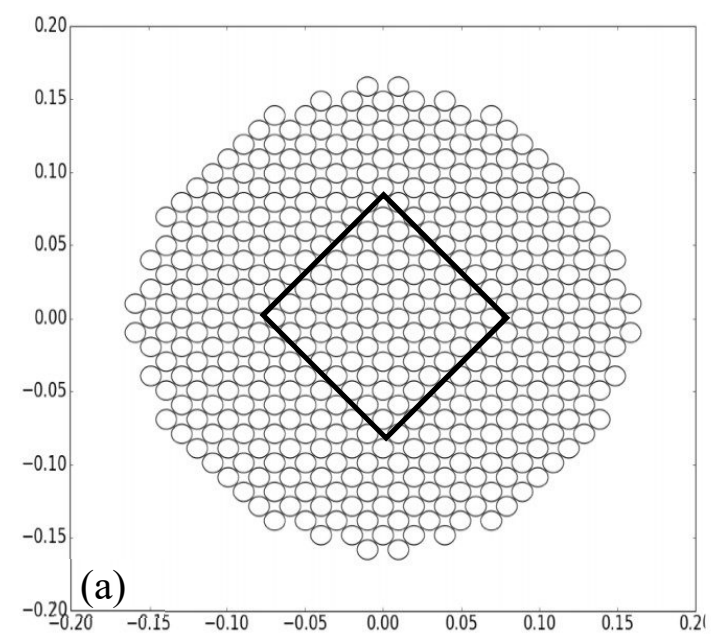

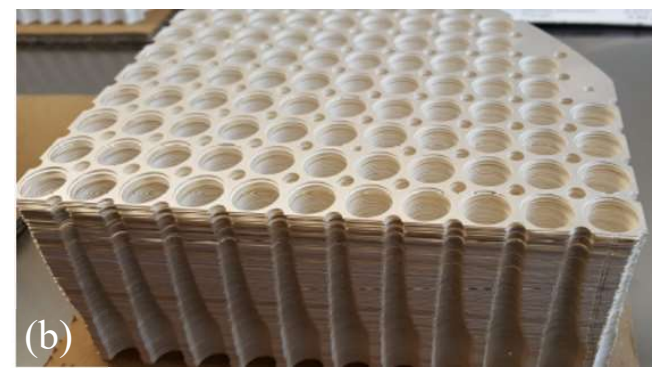

Figure 2 (a) Layout of the 400-element input horn array for the FI. Each horn has an aperture of $6.2 \mathrm{~mm}$ and the horn separation distance is $13.7 \mathrm{~mm}$ The central $8 \times 8$ horns indicated will be used in the TD. (b) Photograph of one quarter of the FI platelet horns (one element of each back-to-back pair).

The advantage of using QUBIC as an interferometer rather than an imager (i.e. with just a clear aperture rather than the array of input horns) is that the redundancy of baselines can be exploited in a self-calibration procedure ${ }^{6,7}$ to correct for systematic effects due to different horn gains and cross-polarization, for example.

\subsection{Design of the QUBIC optical beam combiner}

The design of the optical beam combiner for QUBIC has been described in detail elsewhere ${ }^{9,11}$ and is summarised here. The practical limitation on the size and the number of bolometers that could be produced for the focal plane, as well as the requirement to Nyquist sample the fringes from features on the largest multipole scales, means that the focal length of the combiner is limited to approximately $300 \mathrm{~mm}$. The input array of 400 feedhorns has an aperture diameter of $300 \mathrm{~mm}$ to ensure good sensitivity, making this is a very fast optical system $(\sim f / 1)$. The field-of-view of $12.9^{\circ} \mathrm{FWHM}$ is set by the far-field beam pattern of the horns in the input array. The full combiner must fit into a cryostat of approximately $1 \mathrm{~m}^{3}$. A reflecting rather than refracting design means that the optics can be accurately characterised, particularly in terms of polarisation, and can have a large unobstructed aperture. Taking all these criteria into account, minimising aberrations for a fast, large field-of-view system was then the main driver for the optical design.

We studied several designs for the QUBIC combiner ${ }^{11}$ including compensated classical Cassegrain, Gregorian and Dragonian dual reflectors. Our short focal length and relatively large focal plane ruled out the crossed designs. Finally, a compensated off-axis Gregorian design was chosen that also obeyed the Rusch condition for minimum spillover ${ }^{12}$. A further optimisation of the mirror surfaces was carried out with the aid of commercial ray-tracing software (Zemax, now called OpticStudio ${ }^{13}$ ) to improve the diffraction-limited field-of-view. Figure 4 shows the layout of the combiner which can be compared with the idealised one of Figure 1.

In the next section we describe the optical simulations used to analyse the performance of this combiner design. 

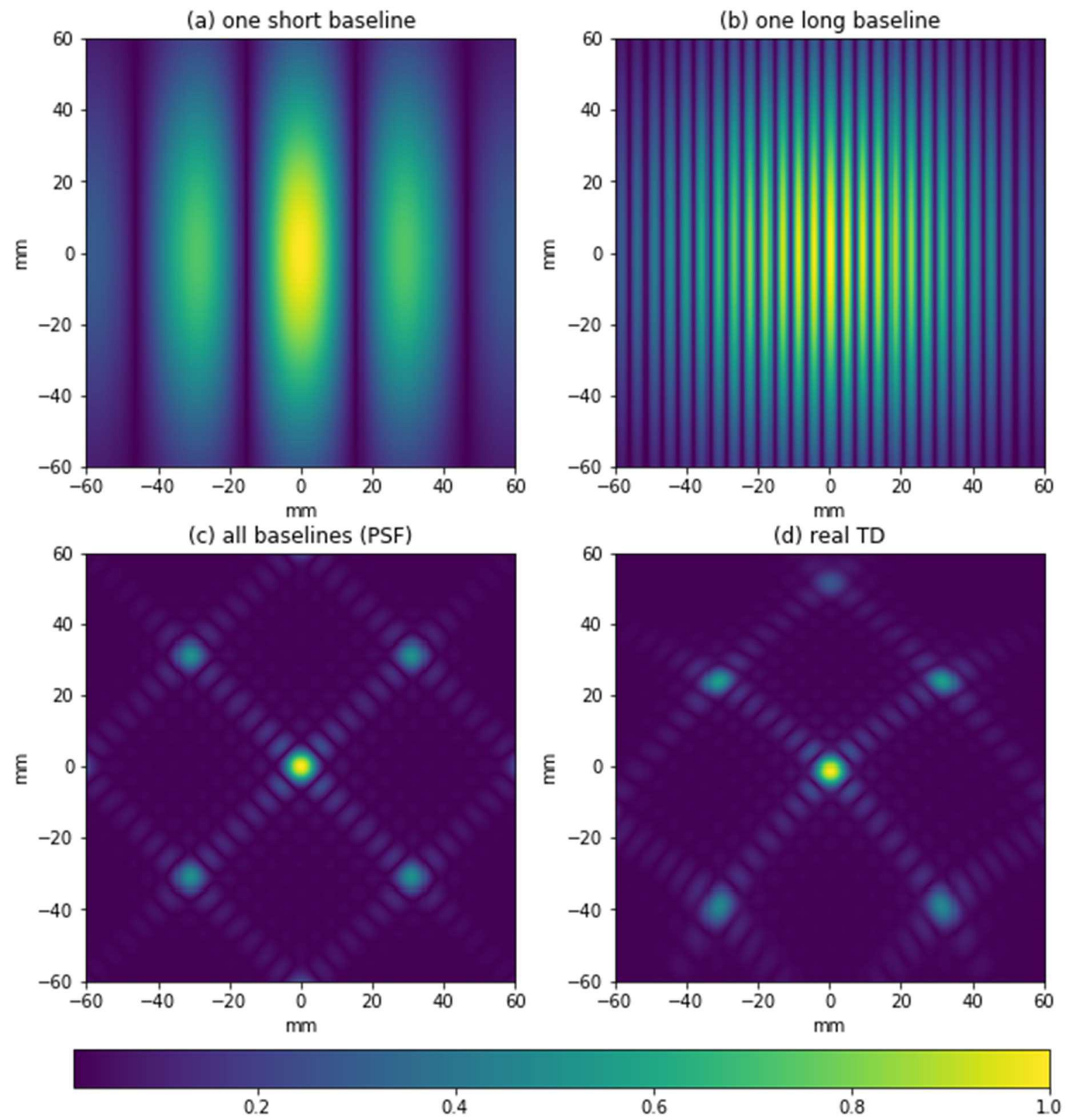

Figure 3. Focal plane images (normalised amplitude) for an imager such as that shown in Figure 1. These images were produced assuming an $8 \times 8$ array of horns, with a spacing of $13.7 \mathrm{~mm}$, producing Gaussian beams (waist radius $w_{0}=3.33 \mathrm{~mm}$ ) at a frequency of $150 \mathrm{GHz}$. The axes of the input horn array were rotated by $45^{\circ}$ with respect to the focal plane (see Figure 2(a)) and the imager had a focal length of $300 \mathrm{~mm}$. The amplitude of the field on the focal plane is shown here for (a) one short baseline $(\sim 10 \lambda)$, (b) one long baseline $(\sim 70 \lambda)$, (c) all baselines simultaneously and (d) all baselines for an aberrating imager. Each plot is normalised to its own peak.

\section{OPTICAL SIMULATIONS}

\subsection{Modelling techniques}

The initial design and final optimisation of the dual-reflector design was carried out using ray-tracing in order to take advantage of the speed and optimisation routines available in the commercial software package Zemax. However, for a 
detailed analysis at the operating frequencies, where component sizes are not very large compared with the wavelength of radiation, techniques that include the effects of diffraction must be used ${ }^{14}$.

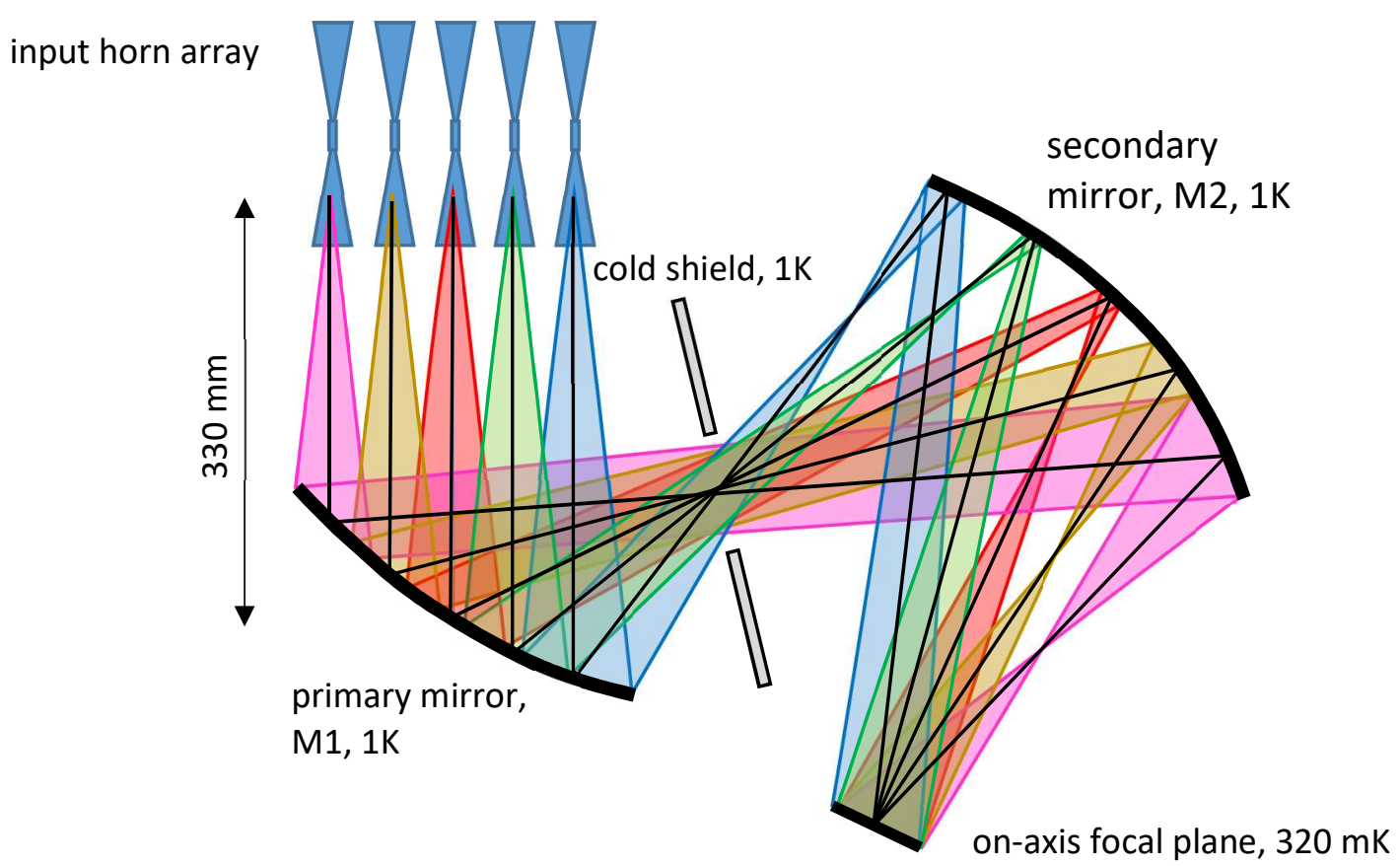

Figure 4. The main components of the QUBIC optical beam combiner. The primary mirror is close to parabolic and shares a focal point with the elliptical secondary. Not shown here are a rotating half-wave plate and filters that will be placed above the input horn array and the dichroic that will direct $220-\mathrm{GHz}$ radiation out of the plane of the paper to a second focal plane. Zemax ray-tracing software was used to draw the beams in this plot.

In our analyses, we start with the beams emitted by the downward facing horn array and propagate them through the optical system i.e. the primary, cold shield aperture (which we call the cold stop), then secondary mirror, dichroic, and on to the focal plane. Initially we use a best-fit Gaussian beam to represent the horn beam and propagate it through an equivalent on-axis system using a Gaussian beam mode analysis and the ABCD technique (see e.g. Goldsmith ${ }^{15}$ ). This is a useful analysis for determining approximate beam sizes in the instrument and on the focal plane. In Figure 5(a) we plot the Gaussian beam radius, as a function of propagation distance through the system, for the central and two edge horns of the FI. The beam sizes predicted by ray tracing (e.g. in Figure 4) are a good approximation to these except where the beams are highly focussed. However, neither gives an accurate picture of the beam profile at these locations (Figure 5(b)); for this we need to use full vector physical optics (PO).

For a more accurate determination of system performance and for the optimisation of the dichroic and cold stop location, a full vector PO analysis of all 400 beams in the combiner was carried out with our custom software MODAL ${ }^{16}$ and the commercially available software GRASP ${ }^{17}$. PO is a very accurate technique provided that the radius of curvature of mirror surfaces is large compared to the wavelength, and it takes into account effects such as diffraction and cross-polarisation. If the field close to the edge of a reflector is significant, so that this PO approximation breaks down, GRASP allows the technique to be supplemented with the physical theory of diffraction (PTD). The original beam emitted from the horns is calculated using a rigorous electromagnetic mode matching technique ${ }^{18}$. Figure 6 shows footprints of the 400 beams on the secondary mirror and cold stop aperture as well as the $64 \mathrm{TD}$ beams on the smaller secondary. Calculating the footprint of the beams at various planes in the system allowed the optimum size and location of components to be determined ${ }^{19}$. The plot on the secondary mirror, in particular shows how some beams are quite focussed at this propagation distance while others are much wider (see also Figure 5(a)) and so all beams need to be modelled at each location of interest. 


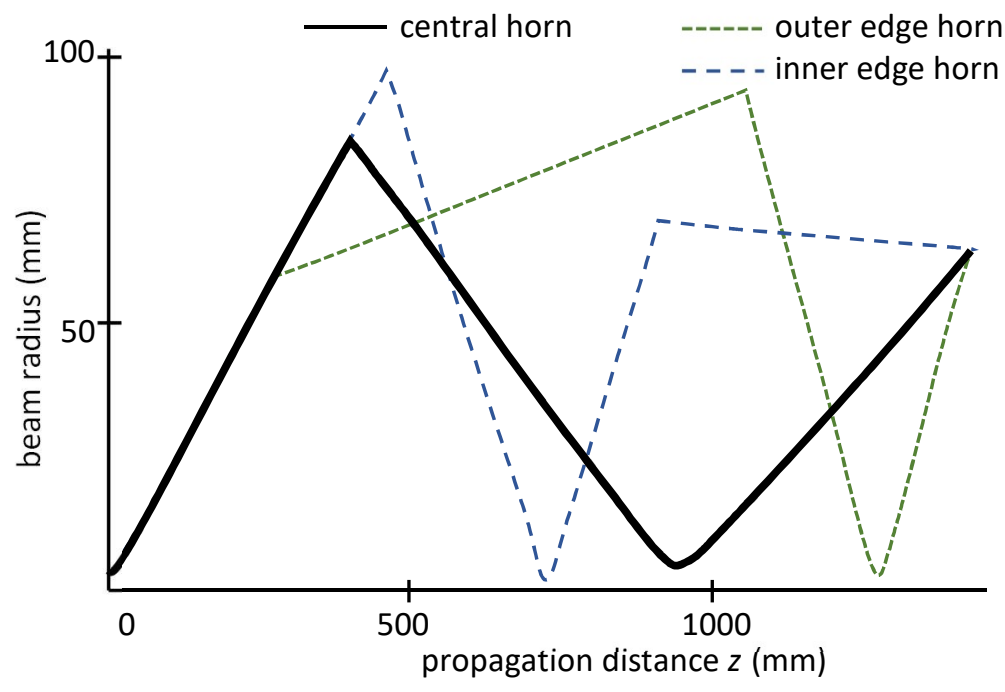

(a)

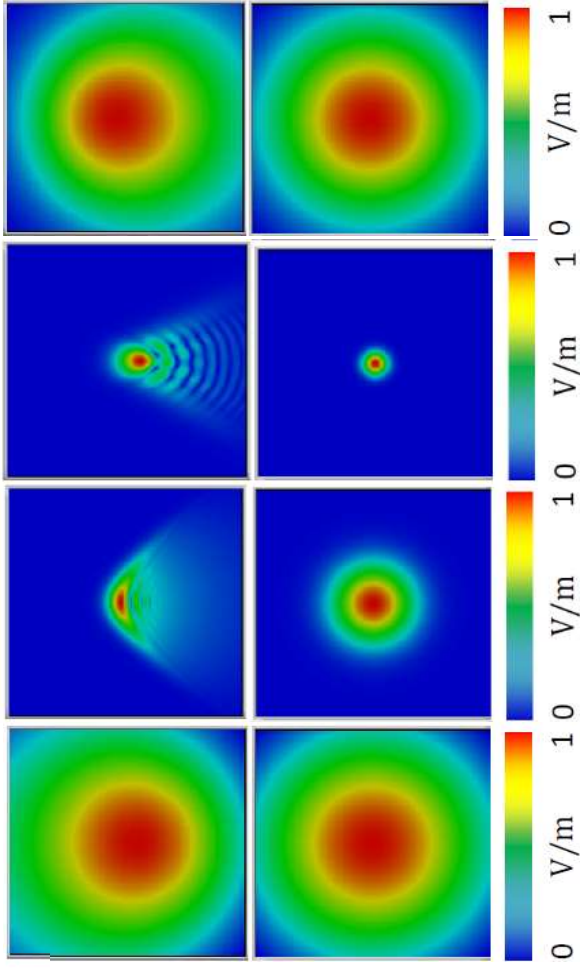

(b)

Figure 5 (a) Best-fit Gaussian beam radius as a function of propagation distance through the QUBIC combiner for beams from a central and two edge horns of the full instrument (left- and right-most horn of Figure 4). The sudden changes in radius occur because of optical components which are located at different distances for each of the horns. (b) The beam from the central horn after the primary mirror $(z=600 \mathrm{~mm}$, top row) secondary mirror ( $z=970 \mathrm{~mm}$, second row), approximately $100 \mathrm{~mm}$ after the secondary mirror $(z=1100 \mathrm{~mm}$, third row $)$ and on the focal plane $(z=1430 \mathrm{~mm}$, bottom row $)$. These were calculated using physical optics (left image on each row) and a Gaussian beam mode approximation (right image on each row). (Adapted from O'Sullivan et al. ${ }^{9}$ and Scully ${ }^{19}$.)

In addition, we have used PO calculations of the beam profiles at successive planes in the combiner to reconstruct 3D models of the beams that can be incorporated into CAD models of the full system ${ }^{20}$ (Figure 6(d)), and allow us to assess potential issues near support structures and additional components. We typically represent the beam using either the $-3 \mathrm{~dB}$ or $-13 \mathrm{~dB}$ power level or else, if the beams are very non-Gaussian, by their $80 \%$ or $95 \%$ encircled power level. This will be especially useful for $220-\mathrm{GHz}$ simulations where there are several independent modes present in the beam ${ }^{9}$ which can be, as a result, very non-Gaussian.

Finally, a stray light analysis was carried out using the non-sequential mode of Zemax. This mode allows tracing of both reflected and transmitted ray paths simultaneously and can model wide-angle surface scattering ${ }^{13}$. Rays were traced both backwards from the focal plane and forwards from the input aperture in order to identify critical and illuminated surfaces, respectively. It was not possible, given our constraints, to design an intermediate, accessible aperture stop (i.e. image of the entrance aperture for an efficient cold stop) in the system but a cold shield will be placed around the focal planes and secondary mirror to reduce background loading on the bare array of detector bolometers. There is a direct ray path from the downward facing horns, through the cold shield aperture and onto the on-axis focal plane but an optical shield around the dichroic will eliminate direct radiation below $16^{\circ}$ and reduce radiation at larger angles (Figure 7). This stray light has been added to our PO model, and we expect it to be negligible compared with our signal. 

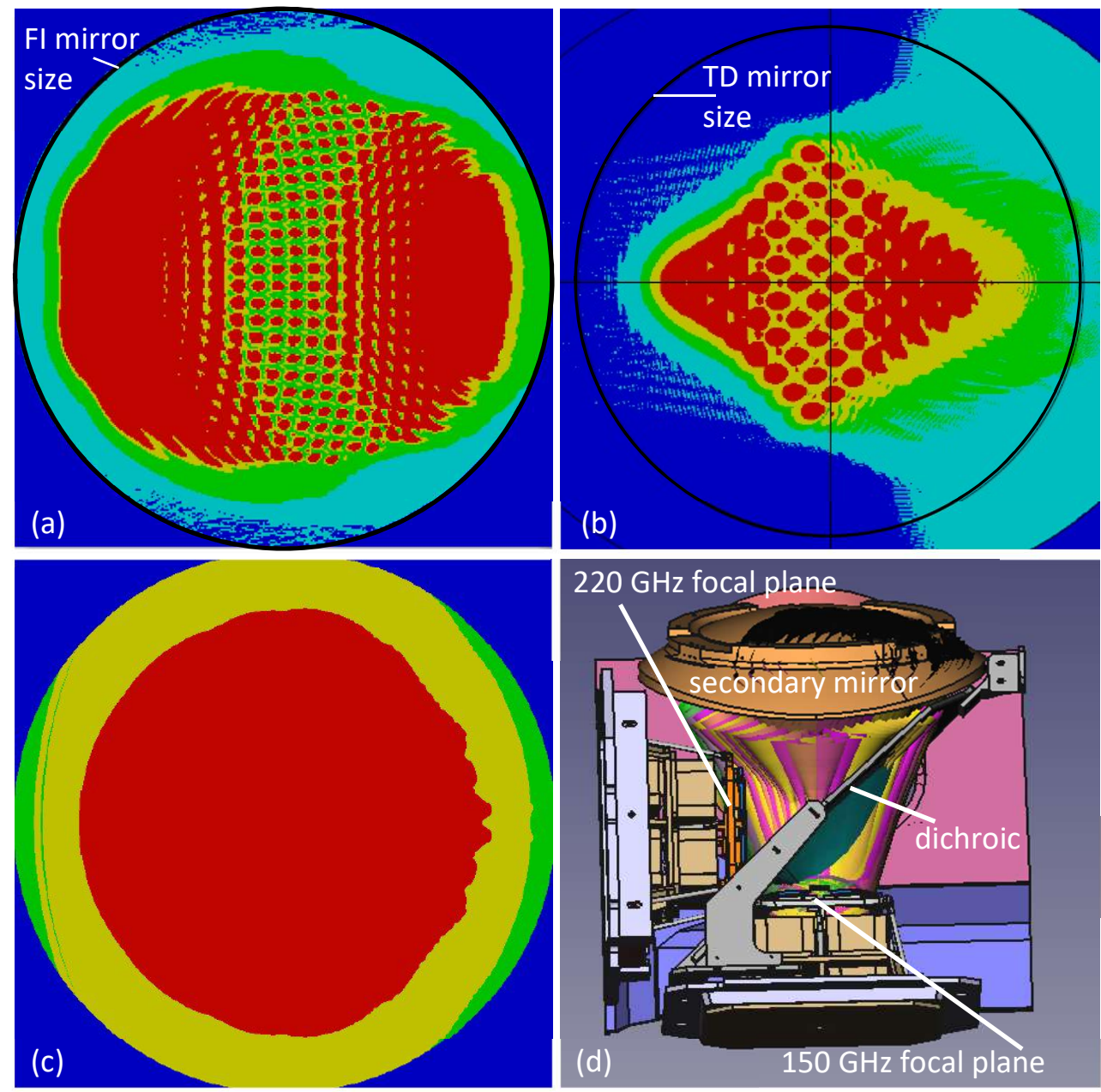

Figure 6. Beam footprints on (a) the FI secondary mirror, (b) the TD secondary mirror (plot is rotated by $45^{\circ}$ compared to (a)) and (c) the cold stop (which is the same size for the FI and TD) ${ }^{15}$. Each beam is coloured so that red corresponds to the region where $72 \%$ of the power of each beam lies, and yellow, green and pale blue to $86 \%, 99 \%$ and $100 \%$, respectively. (d) CAD model of the beam combiner showing the 3D model of the beams (here randomly coloured yellow, pink and green) propagating between the two mirrors.

\subsection{Optical simulations of beam patterns}

We begin the optical simulations by coupling an on-axis plane wave (far-field point source) to a model of our back-toback horn array. The electromagnetic mode-matching technique is then used to generate the aperture field of the downward-facing horns. The 400 (or 64 in the case of the TD) beams are propagated through the system (the two mirrors, cold stop and dichroic aperture) and onto the focal plane using PO. The simulations shown here were all at $150 \mathrm{GHz}$ and show the field on the on-axis focal plane. The focal plane beam patterns can be combined to produce fringe patterns from specific baselines or for all horn open simultaneously.

The combiner requirements mean that some optical aberrations along one plane are unavoidable. Figure 8(a) shows an example of an aberrated fringe pattern and in Figure 8(b) we plot a cut through fringe patterns from 36 baselines equivalent to the one used for Figure 8(a). If the combiner were a perfect imager then these patterns would be identical. Instead the aberrations present are slightly different for each of the baselines and this has the effect of reducing the sensitivity of the QUBIC instrument overall. Figure 3(c) and (d) compare the PSF (all baselines simultaneously) for an ideal and the real (aberrating) QUBIC TD beam combiner. 


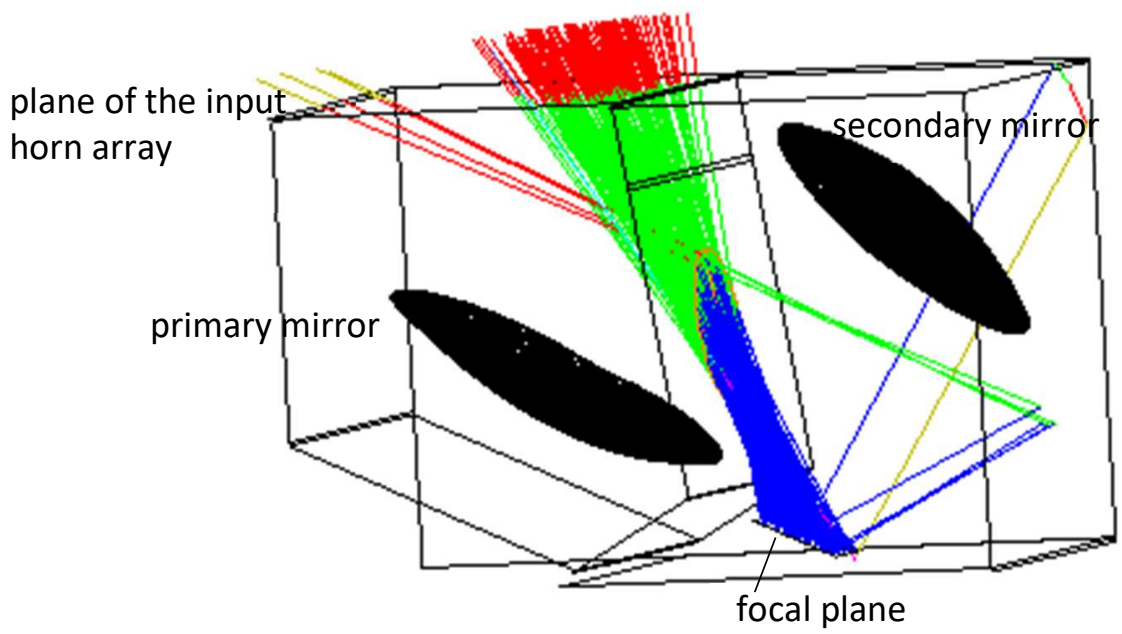

Figure 7. Stray light analysis carried out using Zemax ray-tracing showing a zero-order path from the detector focal plane to the input horn array (radiation from which will be reduced using a shield around the dichroic) and also a (much less significant) first-order path from the detectors to the top of the cryostat.
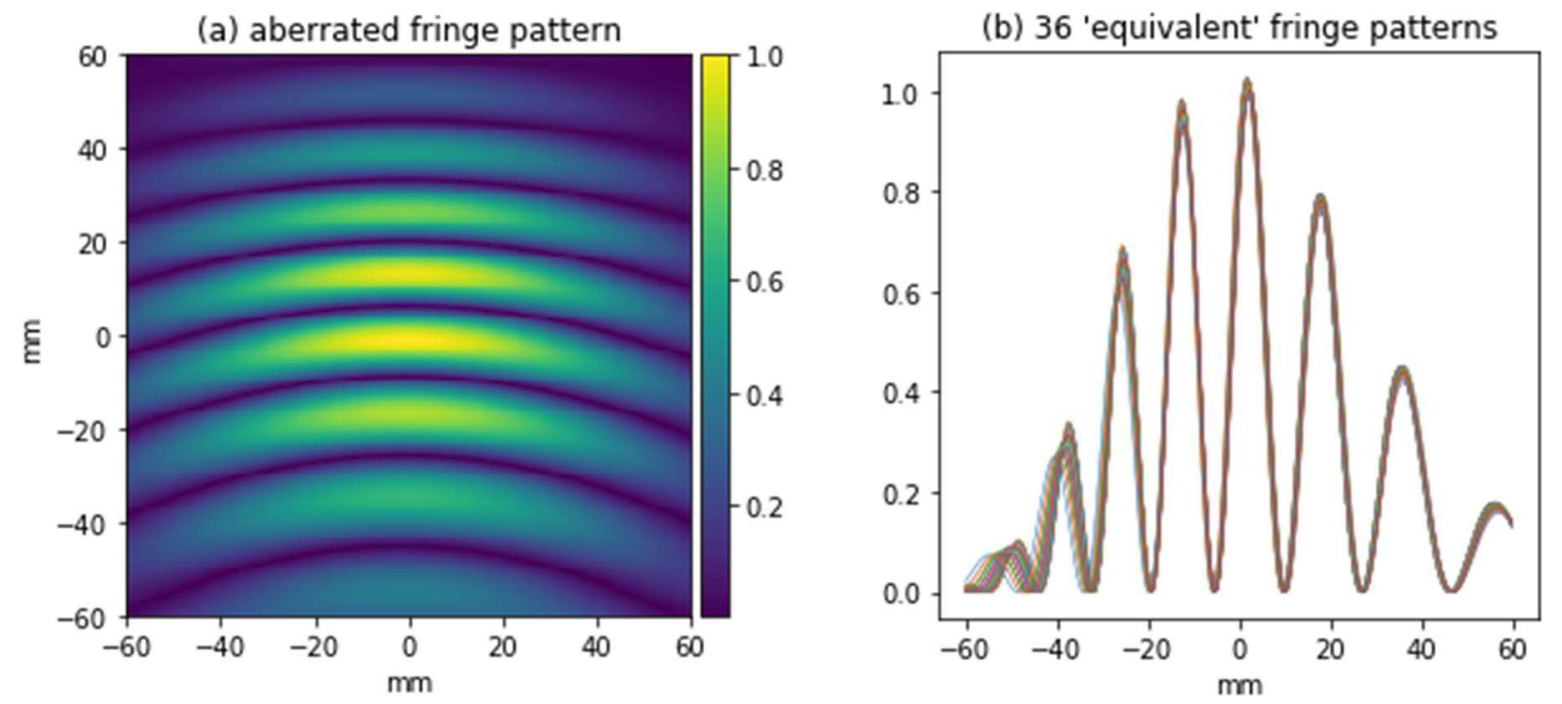

Figure 8. (a) Aberrated fringe pattern resulting from a $\sim 20-\lambda$ baseline along the plane of asymmetry of the combiner (observing an on-axis point source). (b) Vertical cut through 36 fringe patterns each from a $20-\lambda$ (equivalent) baseline.

While figures such as 8(b) give us an indication of the level of aberration present, it is difficult to use them to compare different designs or to predict the effect of the aberrations on the operation of the QUBIC instrument as a whole. For such a comparison we have chosen a figure-of-merit, the window function, which quantifies the sensitivity of QUBIC to different multipoles on the sky. We describe this window function in the following section.

\subsection{The window function}

As described in Section 1.1, the instrument will make synthesised images of the sky in $I, Q$ and $U$ Stokes parameters. An image in total intensity $I$, for example, can be written as

$$
S_{I}\left(p, \bar{n}_{0}, \theta_{0}\right)=\int I(\bar{n}) B_{s}^{p}\left(\bar{n}-\bar{n}_{0}, \theta_{0}\right) d \bar{n}
$$


where $B_{s}^{p}\left(\bar{n}-\bar{n}_{0}, \theta_{0}\right)$ is the synthesised beam pattern for the $p^{\text {th }}$ pixel in the focal plane when the telescope points in the direction $\bar{n}_{0}$ with pitch angle $\theta_{0}$ and $I(\bar{n})$ is the sky intensity in the direction $\bar{n}$ which, as is common in CMB astronomy, can be decomposed into spherical harmonics:

$$
I(\bar{n})=\sum_{l m} a_{l m} Y_{l m}^{*}(\bar{n}) .
$$

where $l$ and $m$ are the usual degree and order number, respectively. As described in Battistelli et al. ${ }^{1}$, we introduce

$$
\beta_{l m}\left(p, \bar{n}_{0}, \theta_{0}\right)=\int B_{s}^{p}\left(\bar{n}-\bar{n}_{0}, \theta_{0}\right) Y_{l m}(\bar{n}) d \bar{n}
$$

so that the synthesised beam pattern for the $p^{\text {th }}$ pixel can be written

$$
B_{s}^{p}\left(\bar{n}-\bar{n}_{0}, \theta_{0}\right)=\sum_{l m} \beta_{l m}\left(p, \bar{n}_{0}, \theta_{0}\right) Y_{l m} .
$$

Making use of the orthogonality of the $Y_{l m}$,

$$
S_{I}\left(p, \bar{n}_{0}, \theta_{0}\right)=\sum_{l m} a_{l m} \beta_{l m}\left(p, \bar{n}_{0}, \theta_{0}\right) .
$$

We introduce the window function

$$
W_{l}\left(p, q, \bar{n}_{k}, \bar{n}_{l}, \theta_{i}, \theta_{j}\right)=\sum_{m} \beta_{l m}\left(p, \bar{n}_{k}, \theta_{i}\right) \beta_{l m}^{*}\left(q, \bar{n}_{l}, \theta_{j}\right) .
$$

which, from Equation (4) above gives a measure of the sensitivity of the instrument to the different multipoles on the sky. We use this window function as our overall figure-of-merit when evaluating the performance of different optical designs or configurations. Similar window functions can be constructed for the other Stokes parameters.

\subsection{Simulated performance of the optical combiner}

Using our PO simulations to find $B_{s}^{p}$, we have calculated the diagonal window function $\left(W_{l}(p, p, \bar{n}, \bar{n}, \theta, \theta)\right.$ where $p$ is a bolometer in the centre of the focal plane) for our aberrating beam combiner and compared it to that of an ideal one. The result, shown in Figure 9, shows that the effect of the aberrations is to reduce the sensitivity of the full instrument by $6 \%$, once integration over the bolometer detector area in the focal plane $(3 \mathrm{~mm} \times 3 \mathrm{~mm})$ is carried out. The loss in sensitivity is due mainly to the aberrations rather than any truncation by the dichroic or cold shield apertures.

In a previous QUBIC design, with a wider horn beam pattern of $14^{\circ} \mathrm{FWHM}$, we estimated a $10 \%$ reduction ${ }^{9,21}$. For the smaller input horn array of the TD the effect of aberrations is less severe and only reduces the sensitivity by about $4 \%$, even without integration over the bolometer area. If we include integration over the bolometers, there is little difference in the window function of the real and an ideal TD (we expect some error from e.g. sampling of the field, especially where the value of the window function is low).

\section{MANUFACTURED MIRRORS}

The TD mirror surfaces as they should be at $1 \mathrm{~K}$ were first designed, and following this a thermal finite element analysis, using the commercial software ANSYS ${ }^{22}$, was used to generate the expected surfaces at 300 (Section 2.2). These $300 \mathrm{~K}$ TD mirrors have been milled from aluminium (6082 alloy) in the University of Milano Bicocca (Figure 10). The mirror surfaces, as manufactured, were then measured in La Sapienza University, Rome, using a Poli Galaxy 3D coordinate measuring machine (CMM) to an accuracy of $5 \mu \mathrm{m}$ with a sampling interval of $5 \mathrm{~mm}$. The measured points were then compared with the nominal $300 \mathrm{~K}$ surfaces (Figure 11).

Finally, in order to incorporate these real mirror surfaces into our optical model, ANSYS was again used to simulate cooling the measured surface back to $1 \mathrm{~K}$. This gave us $\sim 350$ surface points to use in our GRASP PO model. In order to assess the effect of sampling we compared PO models of ideal surfaces defined both analytically and by sampling at 350 
points, again using the diagonal window function as our figure-of-merit. We found that the 350 sampled points were indeed sufficient to accurately describe the mirrors (Figure 12 (a)). Although the surface variations on the real mirrors do produce features in the predicted beams on the focal plane (Figure 13) the manufactured mirrors were also found to be within tolerance, changing the window function by only a small amount $(1 \%$, Figure 12 (b)). Together with the earlier validation of the input horn array ${ }^{8}$ our main optical components were then ready for integration into the cryostat $1 \mathrm{~K}$ box (containing the mirrors, cold shield and the focal plane, Figure 14).
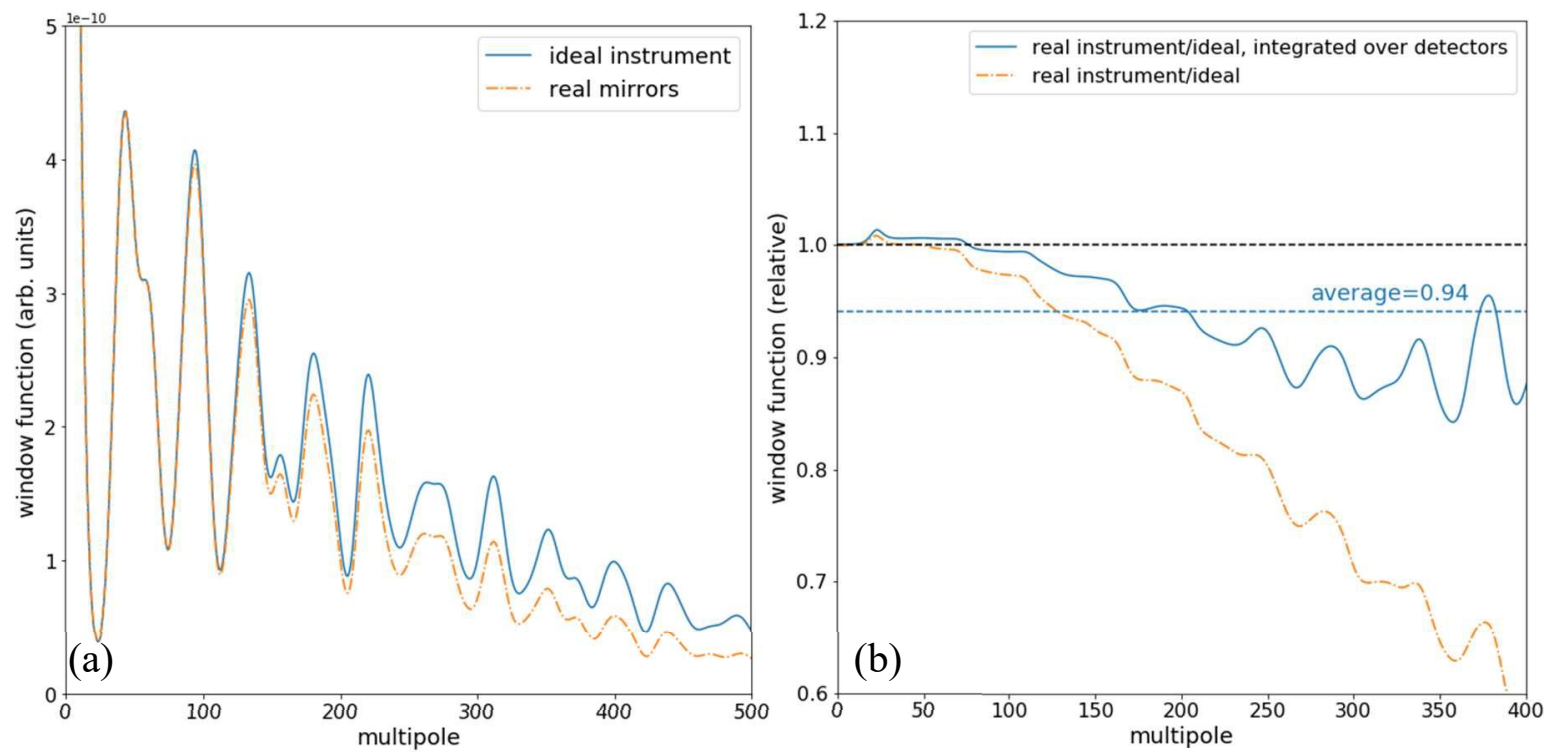

Figure 9. (a) FI Window function for an ideal combiner (e.g. Figure 1) and the real combiner (Figure 4). (b) Relative window function with and without integrating over the bolometer area. (c) Relative window function, with and without integration over bolometer area, for the TD instrument (corresponds to the PSF in Figure 3(d)). Average window function values have been calculated over the multipole range $0-400$ where the sensitivity of the instrument is highest.

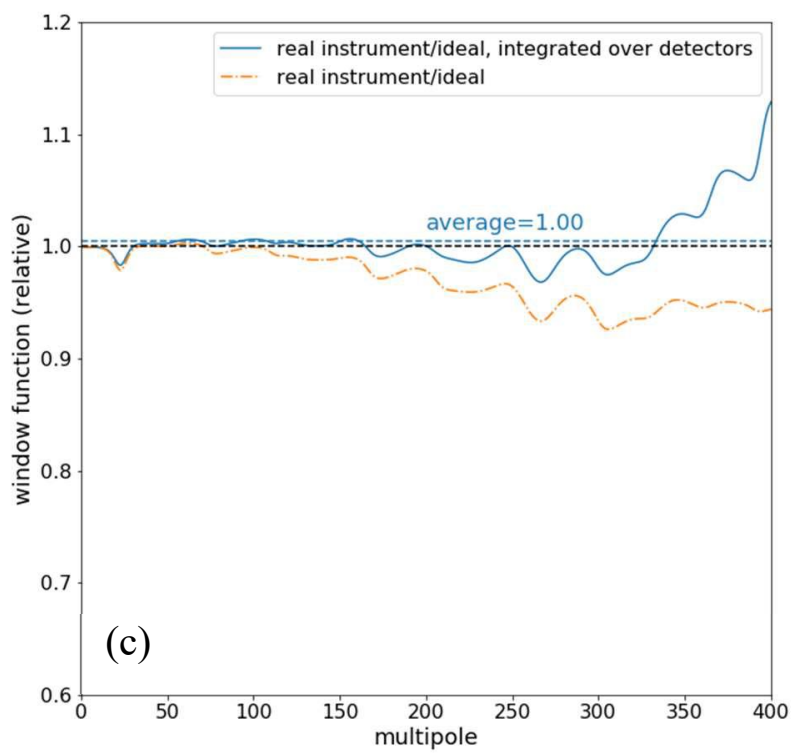



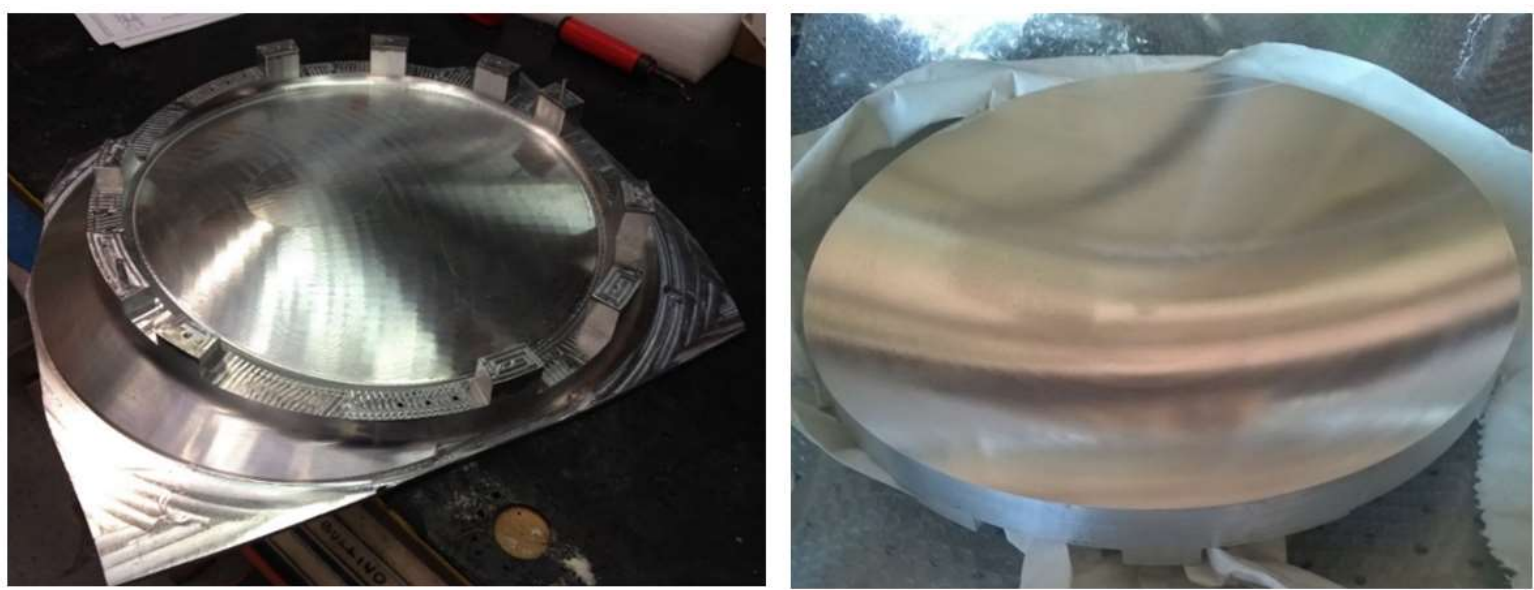

Figure 10. (a) The rear of the TD primary mirror being milled in the University of Milano Bicocca. (b) The front of the TD secondary mirror before polishing.
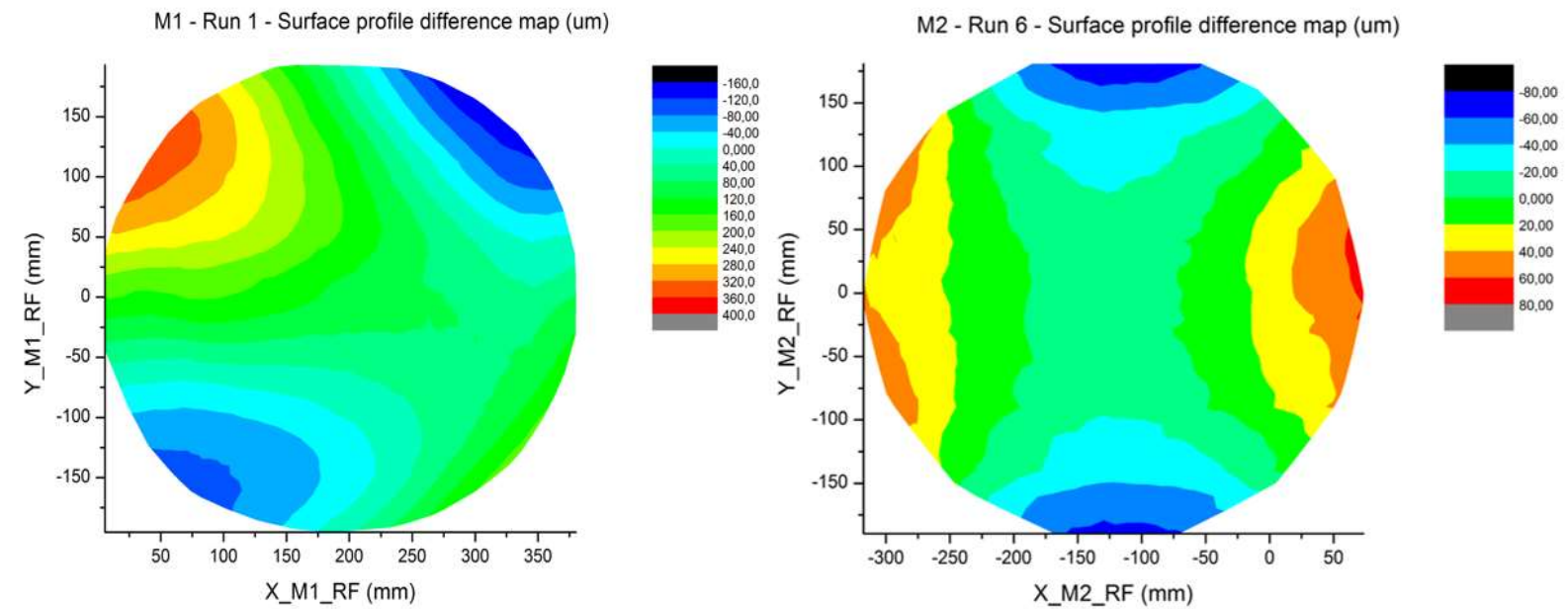

Figure 11. Difference between the measured and nominal mirror surfaces at $300 \mathrm{~K}$. (a) M1 primary mirror (peak-to-valley $511 \mu \mathrm{m}$ ) and (b) M2 secondary mirror (peak-to-valley $137 \mu \mathrm{m}$ ). These figures were generated using a 10-mm sampling of the mirror surfaces.
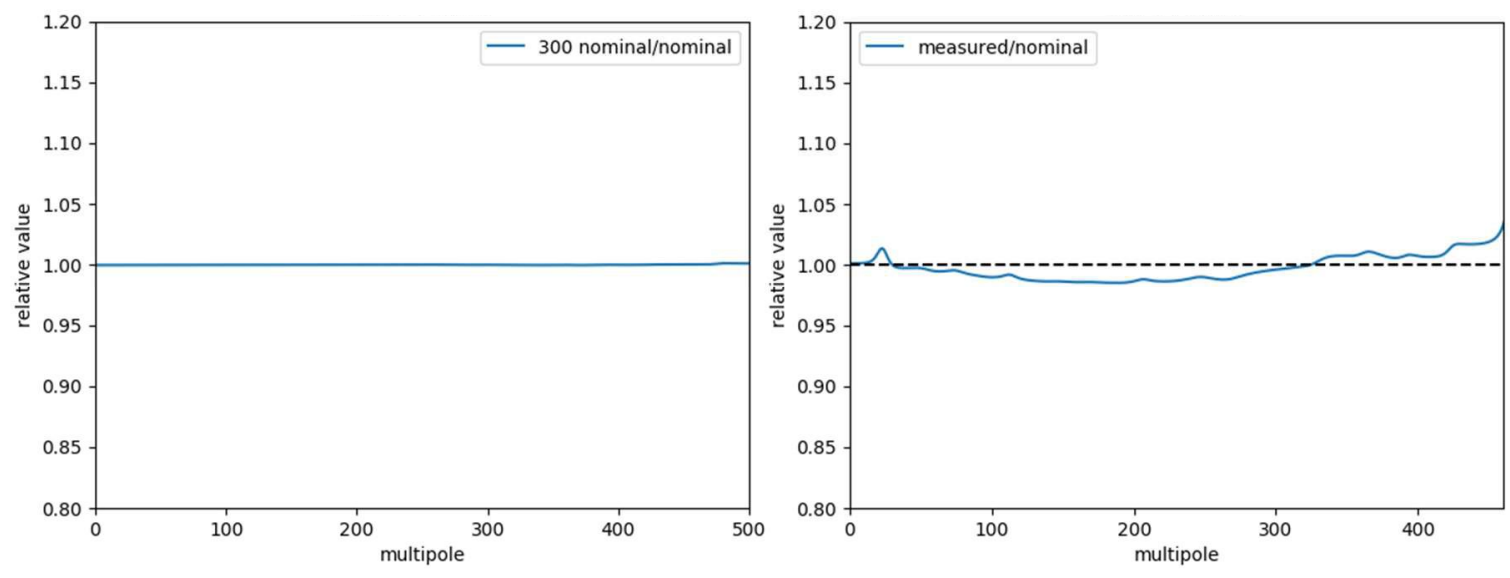

Figure 12 (a) Window function calculated when the QUBIC mirrors are described by 350 sampled points compared with an analytical description. (b) The window function calculated using the measured mirrors compared with the ideal mirrors. 


\section{ALIGNMENT AND TESTING}

A tolerance analysis was carried out to determine the accuracy with which the optical components must be aligned in the cryostat. We considered both displacements and rotations about axes located in the centre of the mirror baseplates (see e.g. Figure 8(a)). Again we have used the overall instrument window function as our figure of merit and want to ensure that it is not decreased by more than about $2 \%$ by mechanical misalignments. The results of this analysis are shown in Table 1 and some examples in Figure 15.
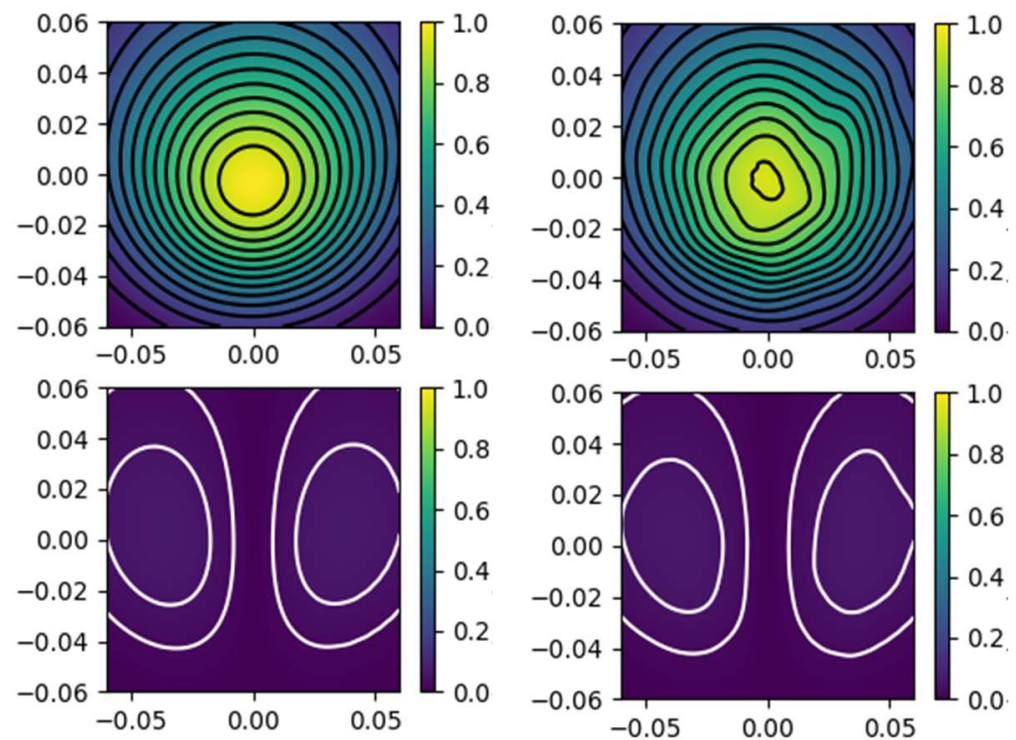

Figure 13. Predicted central horn beam pattern at the focal plane for the ideal (left) and manufactured (right) mirrors. Co- (top row) and cross-polarisation (lower row) are shown, and each pattern is normalised to its own peak. Position in the focal plane is given in meters.

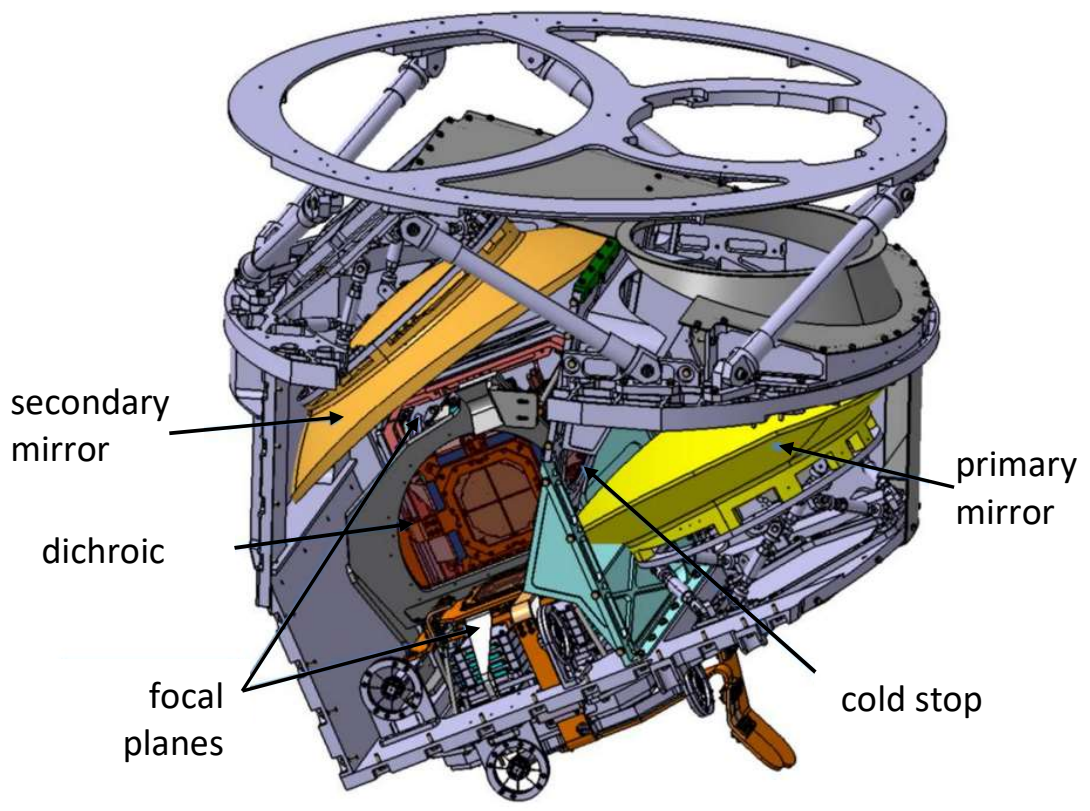

Figure 14 The QUBIC $1 \mathrm{~K}$ box ${ }^{4}$. 
Each of the mirrors was mounted on a hexapod support structure (Figure 14) and its surface measured using a Bras 2018 Sigma $\mathrm{CMM}^{23}$. The CMM arm has an accuracy of $100 \mu \mathrm{m}$ (after correction for the radius of the ruby sphere at the tip of the measuring arm). The hexapods were adjusted until the position of the mirrors matched the specification in Table 1 (Figure 16(a)). The alignment will be further verified using a specially constructed cart and laser measurement system. A He-Ne laser will be moved to the location of the centre of each of the 64 TD horns and the resulting image on a screen placed at the focal plane position will be captured by a CCD camera (Figure 16(b)). Because the mirrors are not of optical quality, a diffuse image is formed and the centroid for each of the 64 laser position is estimated. The predicted location of the laser spot image (at $300 \mathrm{~K}$ ) was found using Zemax ray-tracing simulations. The laser can be tilted and so images will also be made at $\pm 6.5^{\circ}$ along orthogonal axes to match the FWHM of the 150 - GHz beams. Adjustments to mirror location and orientation will be made as necessary.
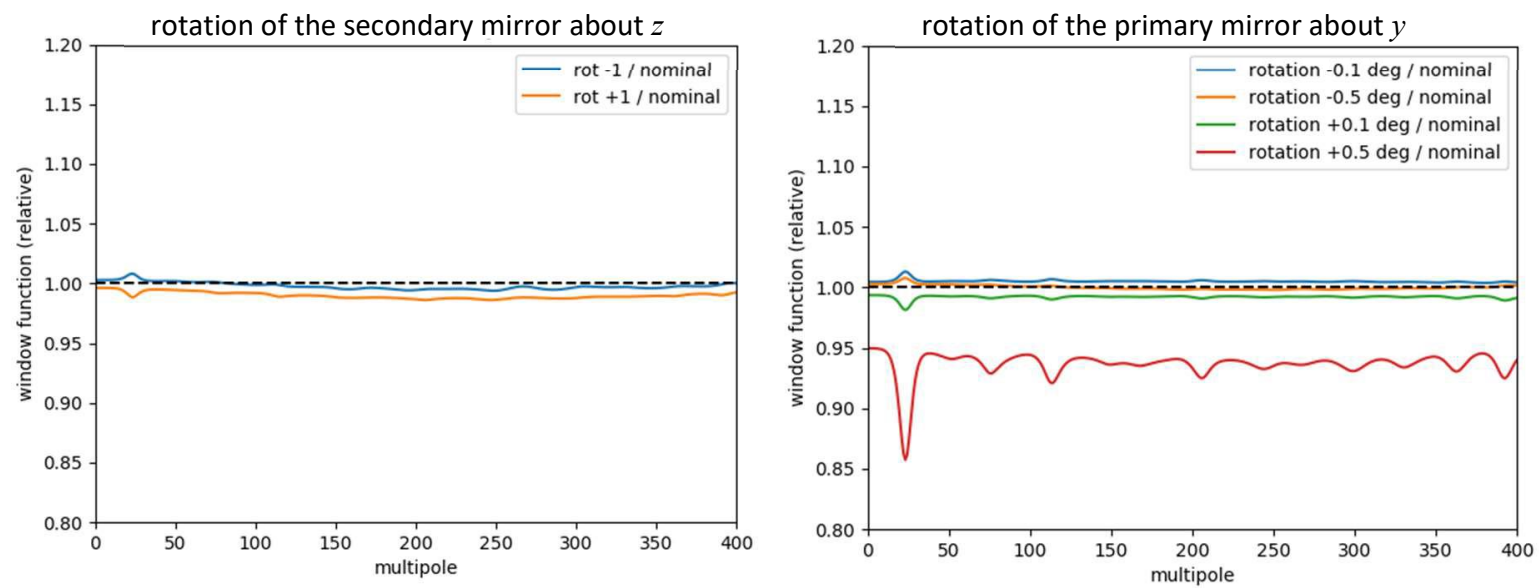

Figure 15 Two examples of window function calculations for the tolerance analysis. Both the secondary (left) and primary mirrors are tolerant to rotations about their local $z$-axis but misalignments must be kept below $0.1^{\circ}$ along the orthogonal axes (right). The window functions were calculated for an on-axis point on the focal plane (rather than integrated over a full detector area) and are shown relative to the window function of an ideal imager.

Table 1 Maximum permitted mis-alignments in the TD mirrors. The axis origins were located in the centre of the mirror baseplate with $x$, and $y$ tangent to the surface and $z$ perpendicular to it.

\begin{tabular}{l|c|c|c} 
component & & translation & rotation \\
\hline \hline primary mirror (M1) & $x$-axis & $\pm 1 \mathrm{~mm}$ & $\pm 0.1^{\circ}$ \\
\hline & $y$-axis & $\pm 1 \mathrm{~mm}$ & $\pm 0.1^{\circ}$ \\
\hline & $z$-axis & $\pm 1 \mathrm{~mm}$ & $\pm 0.5^{\circ}$ \\
\hline secondary mirror (M2) & $x$-axis & $\pm 1 \mathrm{~mm}$ & $\pm 0.1^{\circ}$ \\
\hline & $y$-axis & $\pm 1 \mathrm{~mm}$ & $\pm 0.1^{\circ}$ \\
\hline & $z$-axis & $\pm 1 \mathrm{~mm}$ & $\pm 1^{\circ}$
\end{tabular}

\section{FUTURE PLANS}

An instrument integration and calibration campaign is currently underway in APC and tests at cryogenic temperatures will begin on completion of the room-temperature alignment verification described in the previous section. Of particular importance to the optics will be the inter-calibration of the TES detectors using carbon fibre sources placed inside the 
cryostat. This will also allow a check of the combiner alignment at $1 \mathrm{~K}$ and a monitoring of detector stability. Following this we will make observations of a calibration source in the far-field of the instrument. The aim is to verify the instrument synthesised beam and calibration procedure before shipping to the observing site.

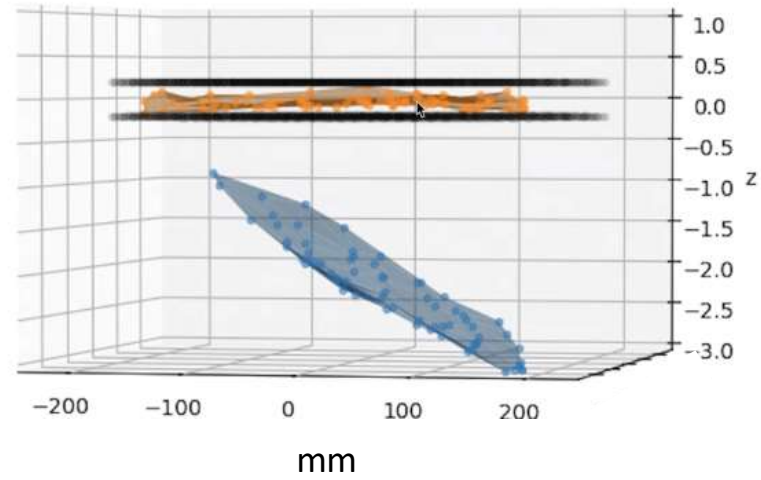

(a)

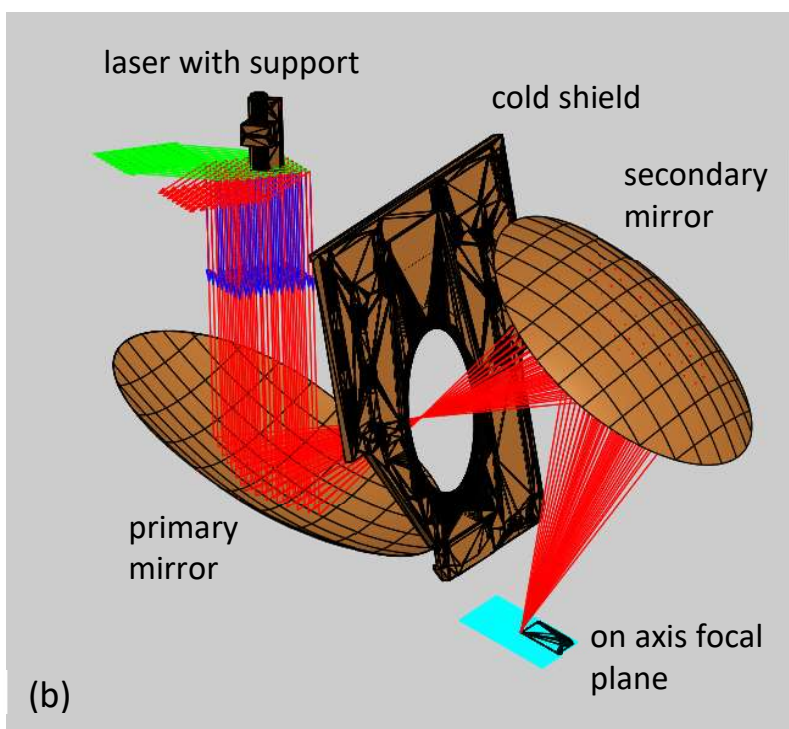

Figure 16 (a) Measured location of the primary mirror surface before (lower, blue) and after (orange) adjustment of the hexapod support legs. The black horizontal lines show the allowed tolerance. (b) GRASP model of the room-temperature alignment test set-up.

The integration of the TD was begun in the spring of 2018. Once upgraded, the first QUBIC module will observe the sky from a site near the city of San Antonio de los Cobres, in the Salta Province of Argentina. The site is at an altitude of 4869 $\mathrm{m}$ above sea level and is located in the Puna de Atacama plateau. This site offers both excellent observing conditions and the accessibility required in the early phases of the project.

\section{ACKNOWLEDGEMENTS}

D. Burke and J.D. Murphy acknowledge funding from the Irish Research Council under the Government of Ireland Postgraduate Scholarship scheme. M. De Leo thanks the Astrophysics Group at the University of Surrey for allowing his continued participation in the QUBIC Collaboration. The APC team acknowledges the financial support of the UnivEarthS Labex program at Sorbonne Paris Cité (ANR-10-LABX-0023 and ANR-11-IDEX-0005-02). INFN in Italy and CNRS/IN2P3 in France are thanked for their funding of the QUBIC project.

\section{REFERENCES}

[1] Battistelli, E., et al., "QUBIC: The QU bolometric interferometer for cosmology”, Astroparticle Physics 34, 705 716 (2011).

[2] Mennella, A., et al., "QUBIC - The Q\&U Bolometric Interferometer for Cosmology - A novel way to look at the polarized Cosmic Microwave Background”, Proc. EPS Conference on High Energy Physics, Venice, Italy (2017). 
[3] O'Sullivan, C., et al. "QUBIC: the Q and U bolometric interferometer for cosmology", these proceedings.

[4] The QUBIC Collaboration, Aumont, J., et al., "QUBIC Technical Design Report”, arXiv:1609.04372v2 (2016).

[5] Zaldarriaga, M. and Seljak, U., "An all-sky analysis of polarization in the microwave background", Phys. Rev. D, 55, p.1830 (1997).

[6] Bigot-Sazy, M.-A.,et al., "Self-calibration: an efficient method to control systematic effects in bolometric interferometry", Astron. \& Astrophys.,550, A59 (2013).

[7] Charlassier, R., et al., "An efficient phaseshifting scheme for bolometric additive interferometry", Astron. \& Astrophys., 497, 963-971 (2009).

[8] Burke, D., et al., "Optical modelling and analysis of the Q and U bolometric interferometer for cosmology", Proc SPIE Photonics West, San Francisco, USA, (to be published 2018)

[9] O'Sullivan, C. et al. "Optical Simulations of the QUBIC Beam Combiner", in Proc. 36th ESA Antenna Workshop on Antennas and RF Systems for Space Science, ESA/ESTEC, Noordwijk, The Netherlands, 6-9 October (2015).

[10] Scully, S., et al., "Optical design and modelling of the QUBIC instrument, a next-generation quasi-optical bolometric interferometer for cosmology”, Proc SPIE Vol. 9914, id. 99142S 15 pp. (2016).

[11] Bennett, D. G., "Design and analysis of a quasi-optical beam combiner for the QUBIC CMB interferometer", PhD Thesis, National University of Ireland, Maynooth (2014).

[12] Rusch W. V. T., et al., "Derivation and application of the equivalent paraboloid for classical offset Cassegrain and Gregorian antennas", IEEE Trans. Antennas and Propagat. 38, (8), 1141 (1990).

[13]Zemax/OpticStudio, Online at https://www.zemax.com/products/opticstudio (April 2018).

[14] O'Sullivan, C., et al., "Far Infra-red Optics Design \& Verification”, International Journal of Infrared and Millimeter Waves, 23 (7), 1029-1045 (2002).

[15] Goldsmith, P.F., [Quasioptical Systems: Gaussian Beam Quasioptical Propagation and Applications], IEEE Press series on microwave technology and techniques (1998).

[16] Gradziel, M. Ł, et al., "Modelling of the optical performance of millimeter-wave instruments in MODAL", Proc. SPIE 6472, 64720D (2007).

[17] GRASP10, TICRA Engineering Consultants. Online http://www.ticra.com/software/grasp/ (May 2018).

[18] Murphy, J. A., et al., "Radiation patterns of multi-moded corrugated horns for far-IR space applications", Infrared Phys. and Tech., 42, 515-528 (2001).

[19] Scully, S., "Quasi-Optical Design and Analysis of a Bolometric Interferometer for Cosmic Microwave Background Experiments", PhD Thesis, National University of Ireland, Maynooth (2016).

[20] Gayer, D., et al., "FreeCAD visualization of realistic 3D physical optics beams within a CAD system-model", Proc. SPIE, Vol. 9914, id. 99142Y 14 pp. (2016).

[21] Marie-Anne Bigot-Sazy, M.-A., “Anisotropie Interférométrie Rayonnement du fond du ciel”, PhD Thesis, Université Paris Diderot, Paris 7, (2013).

[22] ANSYS, https://www.ansys.com/ (April 2018)

[23] Hexagon, www.romer.fr, (April 2018). 